\title{
Reversible changes in grain structure and conductivity in a block copolymer electrolyte
}

Saheli Chakraborty ${ }^{\mathrm{a}}$, Xi Jiang ${ }^{\mathrm{b}}$, Zach J. Hoffman ${ }^{\mathrm{c}, \mathrm{d}}$, Gurmukh K. Sethid ${ }^{\mathrm{d}, \mathrm{e}}$, Chenhui Zhu ${ }^{\mathrm{f}}$, Nitash P. Balsara*a,c,d,e Irune Villaluenga*e

${ }^{a}$ Energy Storage \& Distributed Resources Division, Lawrence Berkeley National Laboratory, Berkeley, California 94720, USA

${ }^{b}$ Materials Sciences Division, Lawrence Berkeley National Laboratory, Berkeley, California 94720, USA

'Joint Center for Energy Storage Research (JCESR), Lawrence Berkeley National Laboratory, Berkeley, California 94720, USA

dDepartment of Materials Science and Engineering, University of California, Berkeley, California 94720, USA

eDepartment of Chemical and Biomolecular Engineering, University of California, Berkeley, California 94720, USA

${ }^{f}$ Advanced Light Source, Lawrence Berkeley National Lab, California 94720, USA

*Correspondence to: nbalsara@berkeley.edu and irvillaluenga@gmail.com 


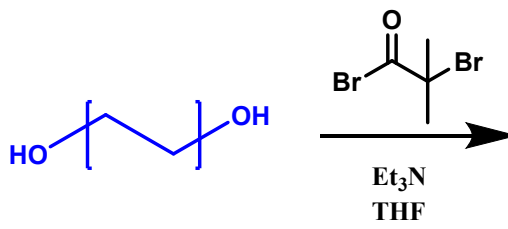

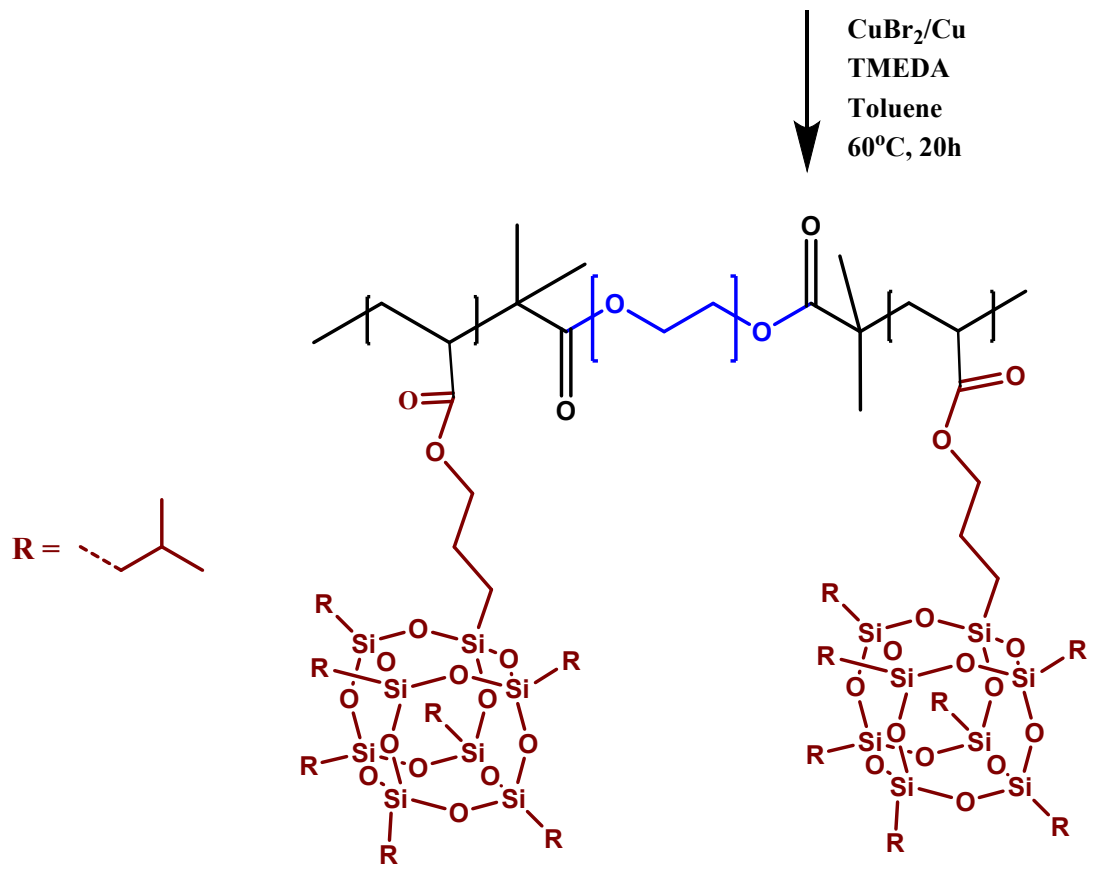

Figure S1. Schematic representation of synthesis of POSS-PEO-POSS 


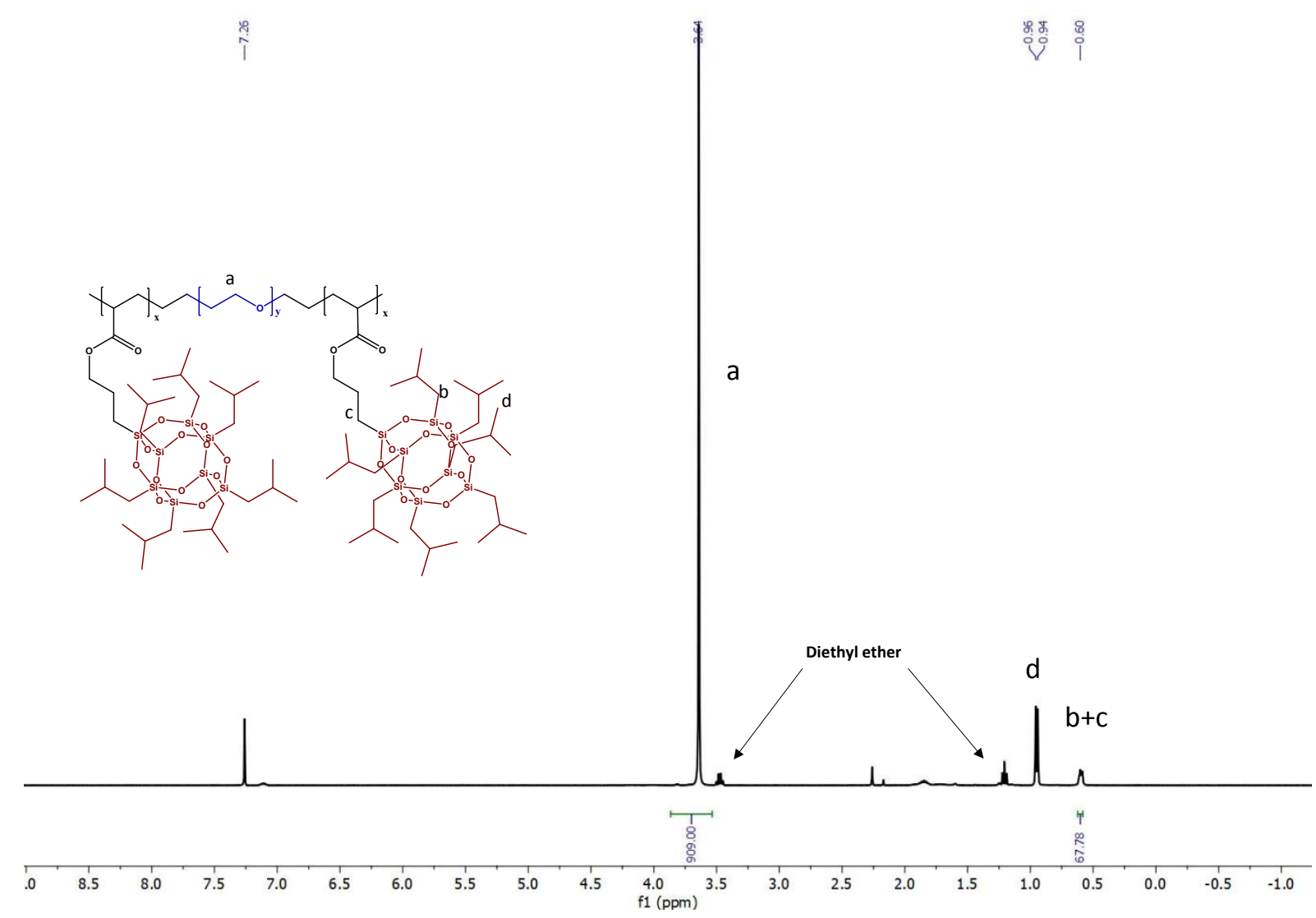

Figure S2. 1H NMR spectra of POSS-PEO-POSS is shown. The sharp peak at $3.65 \mathrm{ppm}$ is due to PEO chains and peak at 0.94 and $0.59 \mathrm{ppm}$ are due to the isobutyl chains of POSS segment as indicated in the figure. The NMR calculation indicates that there are 4 POSS chains per PEO molecule. 


\section{GPC of polymer:}

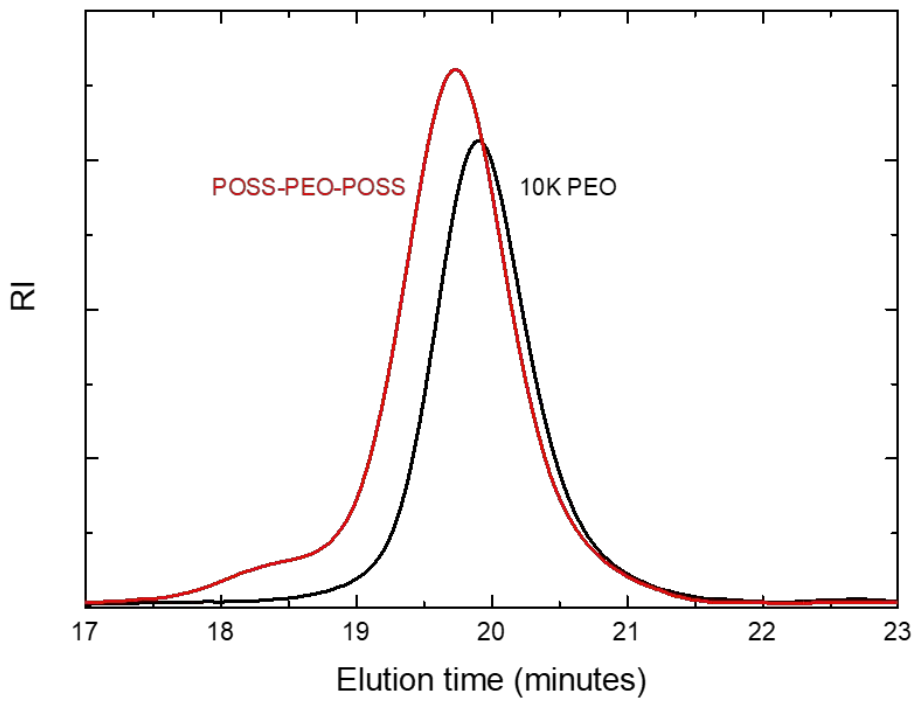

Figure S3. Gel permeation chromatographic data on POSS-PEO-POSS and PEO ATRP initiator. 


\section{Calculation of volume fraction of the electrolyte}

The volume fraction, $f_{E O / L i T F S I}$, of the salty PEO domain is determined based on the following equation

$f_{E O / L i T F S I}=\frac{v_{E O}+r v_{L i T F S}}{v_{E O}+r v_{\text {LiTFSI }}+\frac{M_{P P O S S} M_{E O} O}{M_{P O S S} M_{P E O}} v_{P O S S}}$

here, $\mathrm{M}_{\mathrm{POSS}}$ and $\mathrm{M}_{\mathrm{EO}}$ are the molar mass of POSS (929.61 g/mol) and EO monomer units (44.05 g/mol), respectively; $v_{\mathrm{EO}}, v_{\mathrm{POSS}}, v_{\mathrm{LiTFS}}$ are the molar volumes of ethylene oxide monomer units, POSS monomer units, and LiTFSI, respectively and are calculated based on the following equation,

$v_{i}=\frac{M_{i}}{\rho_{i}}$

where, $\mathrm{M}_{\mathrm{i}}$ and $\rho_{i}$ are the molar masses and density of species i, respectively. $\rho_{\text {LiTFSI }}=2.392 \mathrm{~g} / \mathrm{cm}^{3}$; $\mathrm{M}_{\text {LiTFSI }}=287.09 \mathrm{~g} / \mathrm{mol} ;(1) \rho_{\text {PEO }}=1.128 \mathrm{~g} / \mathrm{cm}^{3}$, and $\rho_{\text {POSS }}=1.30 \mathrm{~g} / \mathrm{cm}^{3}$ at $90{ }^{\circ} \mathrm{C}$ determined by the procedure outlined in ref (2) measuring the weight of a known volume of the diblock copolymer. We considered that the value of $\rho_{\text {POSS }}=1.30 \mathrm{~g} / \mathrm{cm}^{3}$ holds for polymerized POSS in the triblock copolymer.

The volume fraction of PEO domain $f_{\mathrm{PEO}}$ in neat POSS-PEO-POSS (2-10-2) is 0.76 . The volume fraction of salty PEO phase $\left(f_{\mathrm{PEO} / \mathrm{LiTFSI}}\right)$ in the electrolyte $(r=0.02)$ is 0.77 . 


\section{Determination of the scattering invariant $S$}

The scattering invariant, $S$, is given by the equation.

$S=\int_{q 1}^{q 2} I q^{2} d q$

The scattering invariant was computed using Igor Pro software by plotting $I q^{2}$ vs $q$, as shown in figure S4. The intensity of scattering peak was calculated by subtracting the baseline, fit near the primary peak $q^{*}$. The peaks were fitted to a Gaussian function to determine the area.

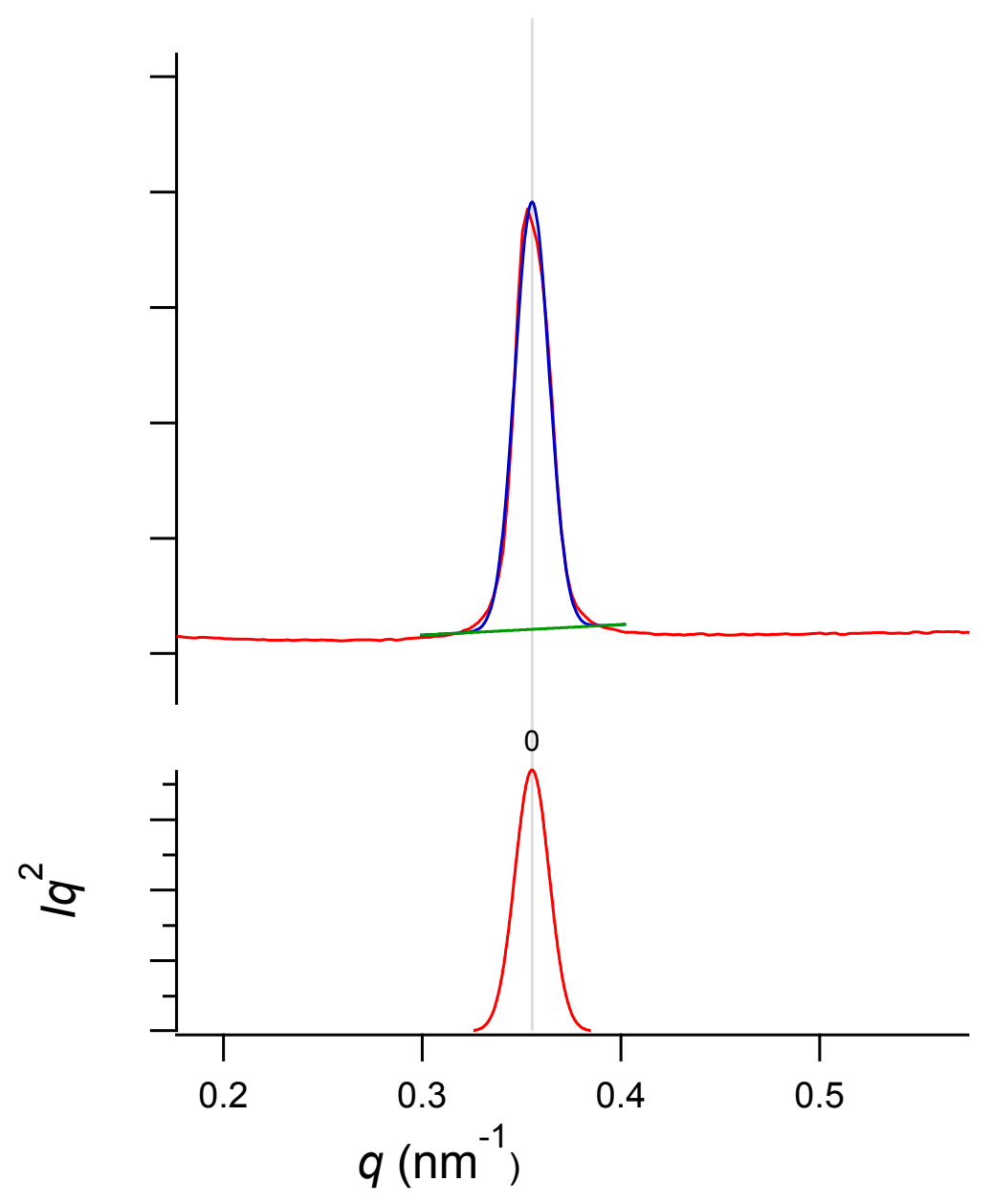

Figure S4. $I q^{2}$ is plotted against scattering vector $q$ for the electrolyte at $103{ }^{\circ} \mathrm{C}$ (bottom plot). The curve was fitted to a Gaussian function (top plot, blue line indicates the fit). The blue line in the top image indicates the fit. The area was computed between 0.3 to $0.45 \mathrm{~nm}^{-1}$ using Igor Pro software. 


\section{Invariant for neat copolymer:}

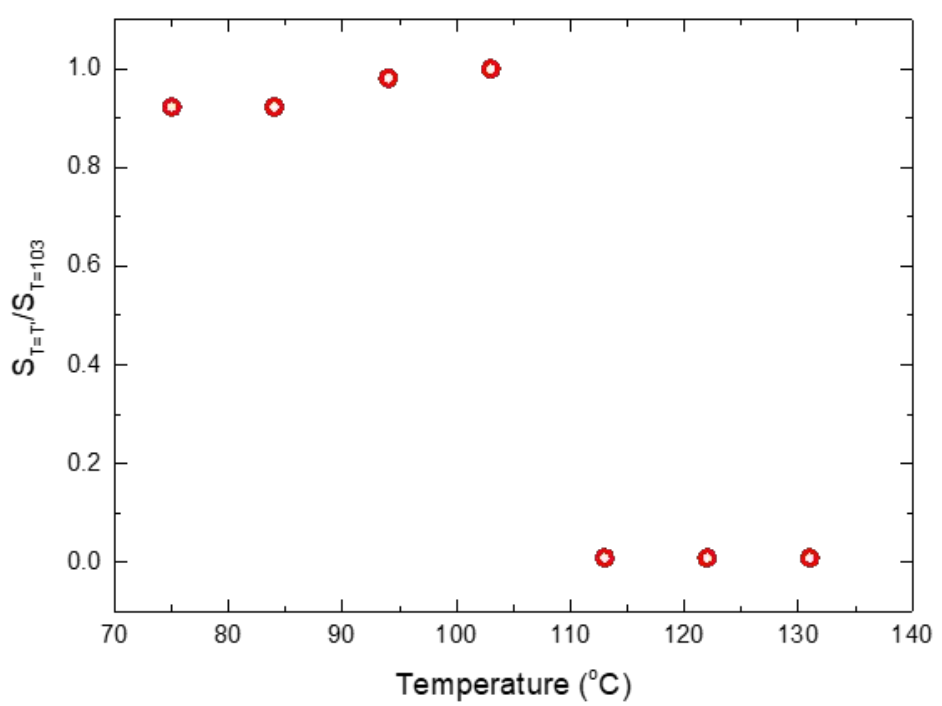

Figure S5. The ratio of scattering invariant $S_{T=T^{\prime}} / S_{T=103}$, where $T^{\prime}$ is the temperature of choice, is plotted against the temperature for the neat POSS-PEO-POSS. The value was computed using Igor Pro software by integrating the primary scattering peak $q^{*}$ over the range from 0.35 to $0.45 \mathrm{~nm}^{-1}$.

\section{Calculation of density of POSS at $113^{\circ} \mathrm{C}$ :}

$\frac{\text { Scattering contrast between } E O \text { and POSS at } 90^{\circ} \mathrm{C}}{\text { Scattering contrast between } E O \text { and POSS at } 113^{\circ} \mathrm{C}}=\frac{\text { Scattering invariant at } 94^{\circ} \mathrm{C}}{\text { Scattering invariant at } 113^{\circ} \mathrm{C}}=115$

Scattering contrast at any temperature $=v_{r e f}\left(B_{P E O}-B_{P O S S}\right)^{2}$

Where $B_{\mathrm{i}}$ is given by,

$$
B_{i}=\frac{r_{e} n_{i} N_{A v g} \rho_{i}}{M_{i}}
$$

Here, $v_{r e f}$ is the reference volume given by $0.1 \mathrm{~nm}^{3}, r_{e}$ is the cross-sectional scattering radius of a free electron, $N_{A v g}$ is Avogadro's number, $n_{i}$ is the number of electrons per $i, \rho_{i}$ is the density at any given temperature and $M_{i}$ is the molar mass of $i$.

$\rho_{\text {POSS }}$ at $90{ }^{\circ} \mathrm{C}=1.3 \mathrm{~g} \mathrm{~cm}^{-3}$

$\rho_{P E O}$ at $90{ }^{\circ} \mathrm{C}=1.128 \mathrm{~g} \mathrm{~cm}^{-3}$, and at $113{ }^{\circ} \mathrm{C}=1.114$

Equation 1 holds when $\rho_{\text {POSS }}$ at $113{ }^{\circ} \mathrm{C}=1.14$ 
DSC thermogram of neat copolymer:

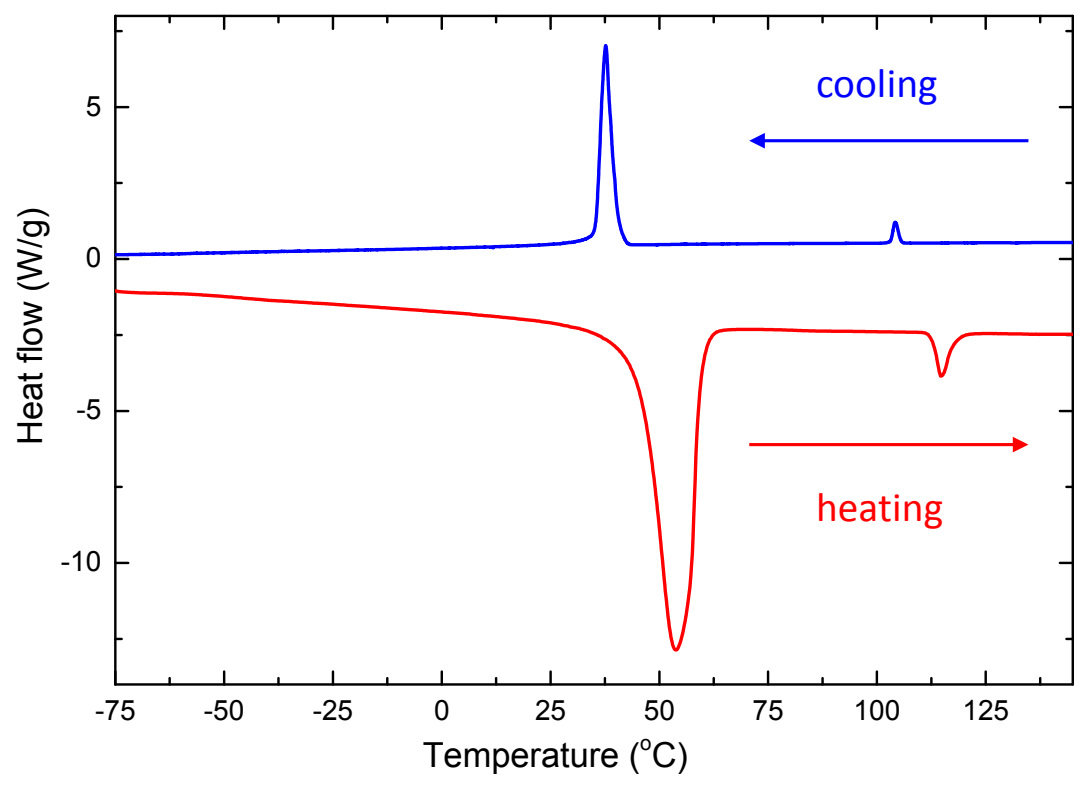

Figure S6. Differential Scanning Calorimetry thermogram of the neat POSS-PEO-POSS. The copolymer exhibits melting peak for PEO domain as well as the POSS domain. 


\section{Rheology of neat copolymer:}

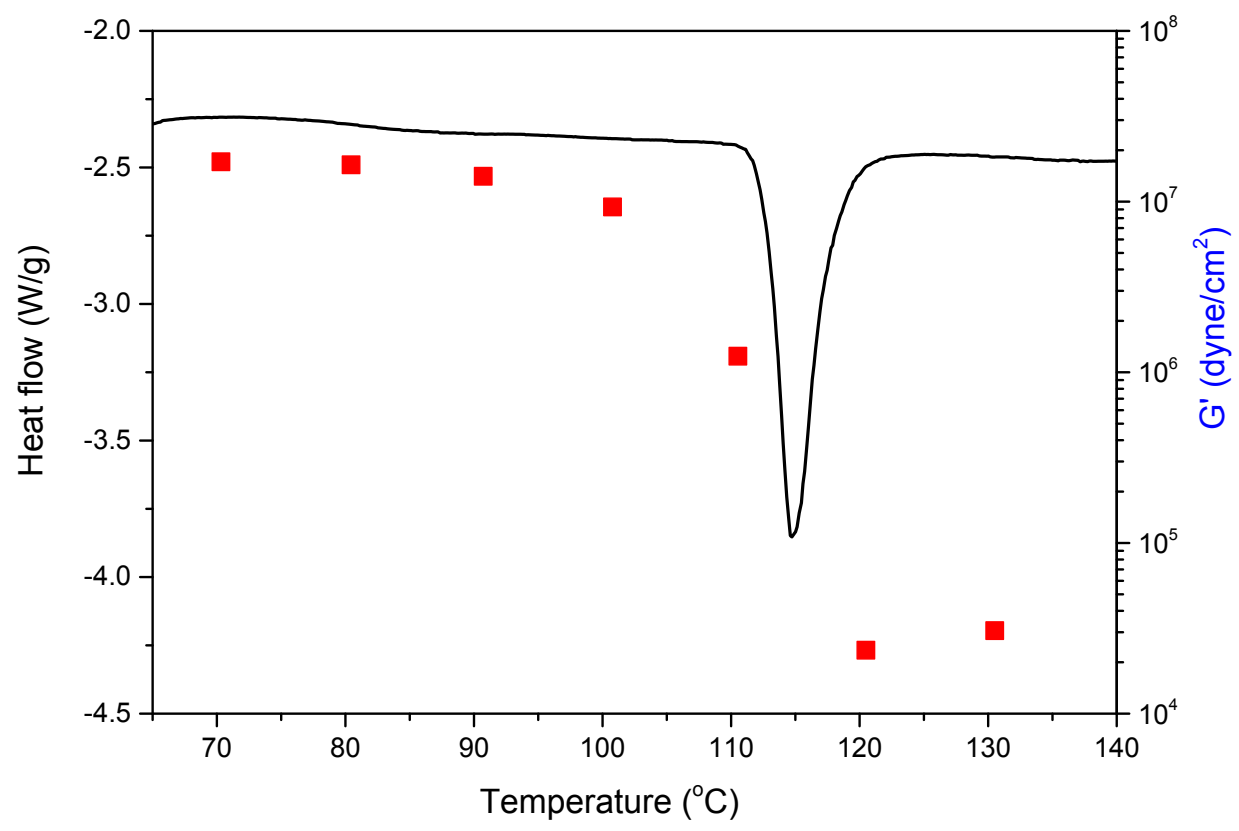

Figure S7. The DSC thermogram is coplotted with the storage modulus $\mathrm{G}^{\prime}$ of the neat POSS-PEOPOSS. The storage modulus decreases sharply from $10^{7}$ to $10^{4}$ dyne $/ \mathrm{cm}^{2}$ at $120{ }^{\circ} \mathrm{C}$ indicating melting of POSS domain. 
DSC thermogram of POSS homopolymer:

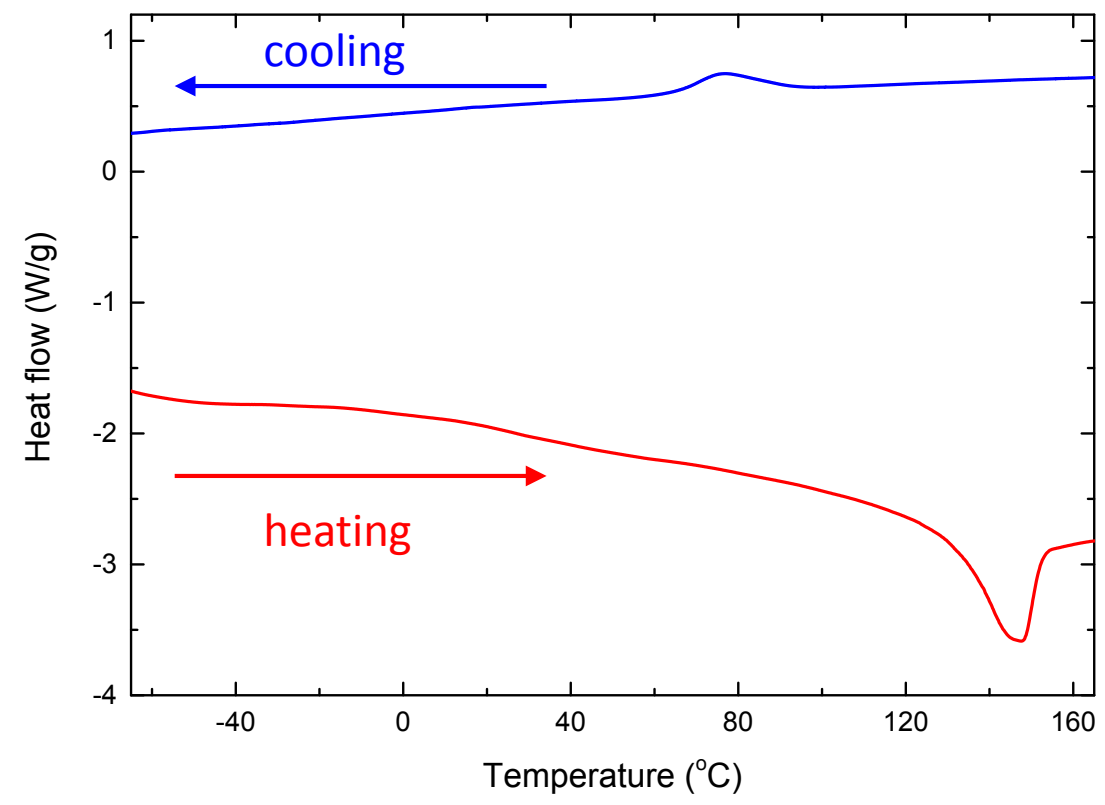

Figure S8. DSC thermogram of POSS-homopolymer. The heating curve indicates melting peak at 147 ${ }^{\circ} \mathrm{C}$. 
WAXS pattern of neat copolymer:

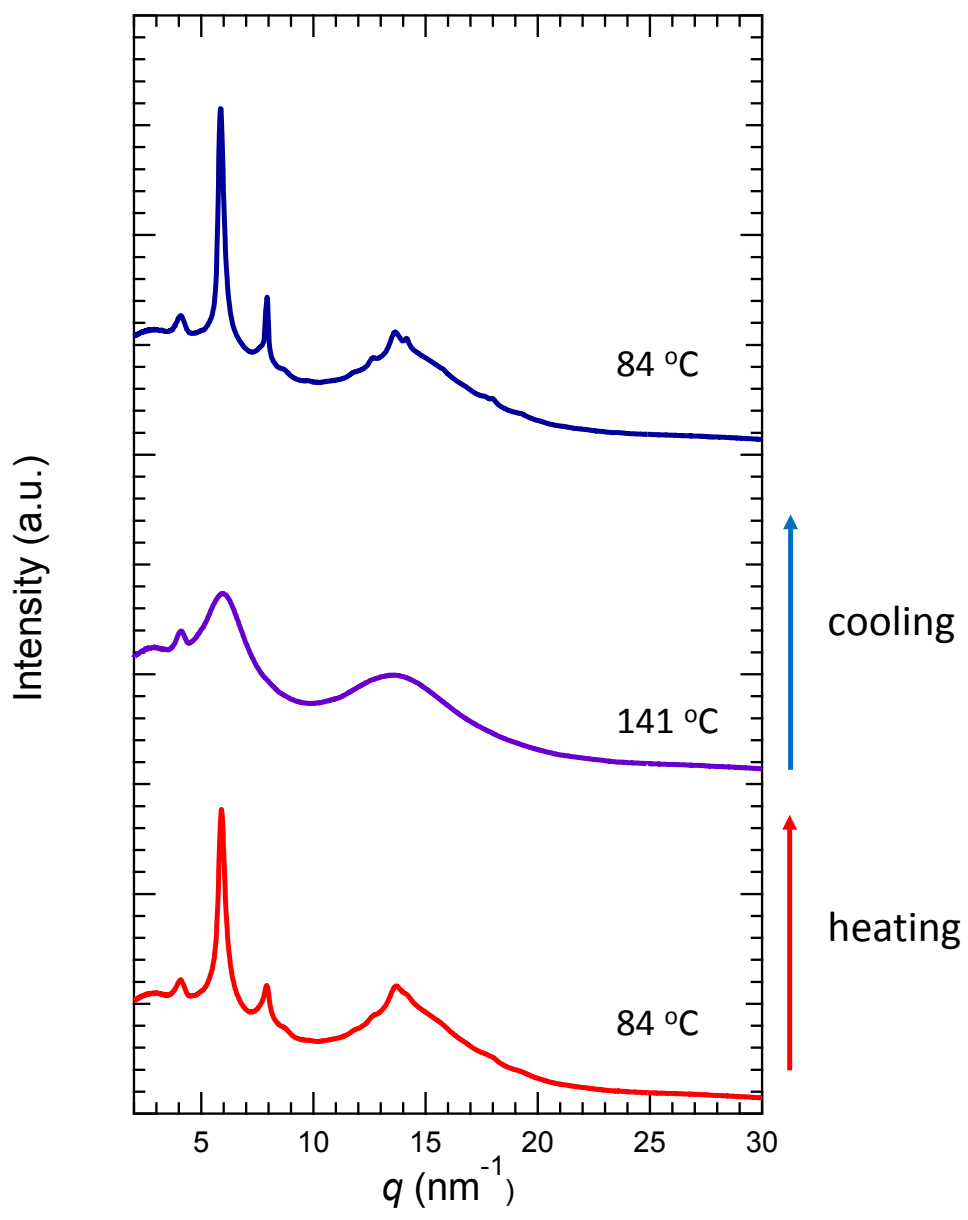

Figure S9. The scattering intensity is plotted against the scattering vector, $q$, for three temperatures for the neat POSS-PEO-POSS copolymer. The WAXS pattern indicates the melting and recrystallization of POSS microphases. The sharp peak at $4 \mathrm{~nm}^{-1}$ is due to kapton window (Figure S10). 
WAXS pattern of POSS homopolymer:

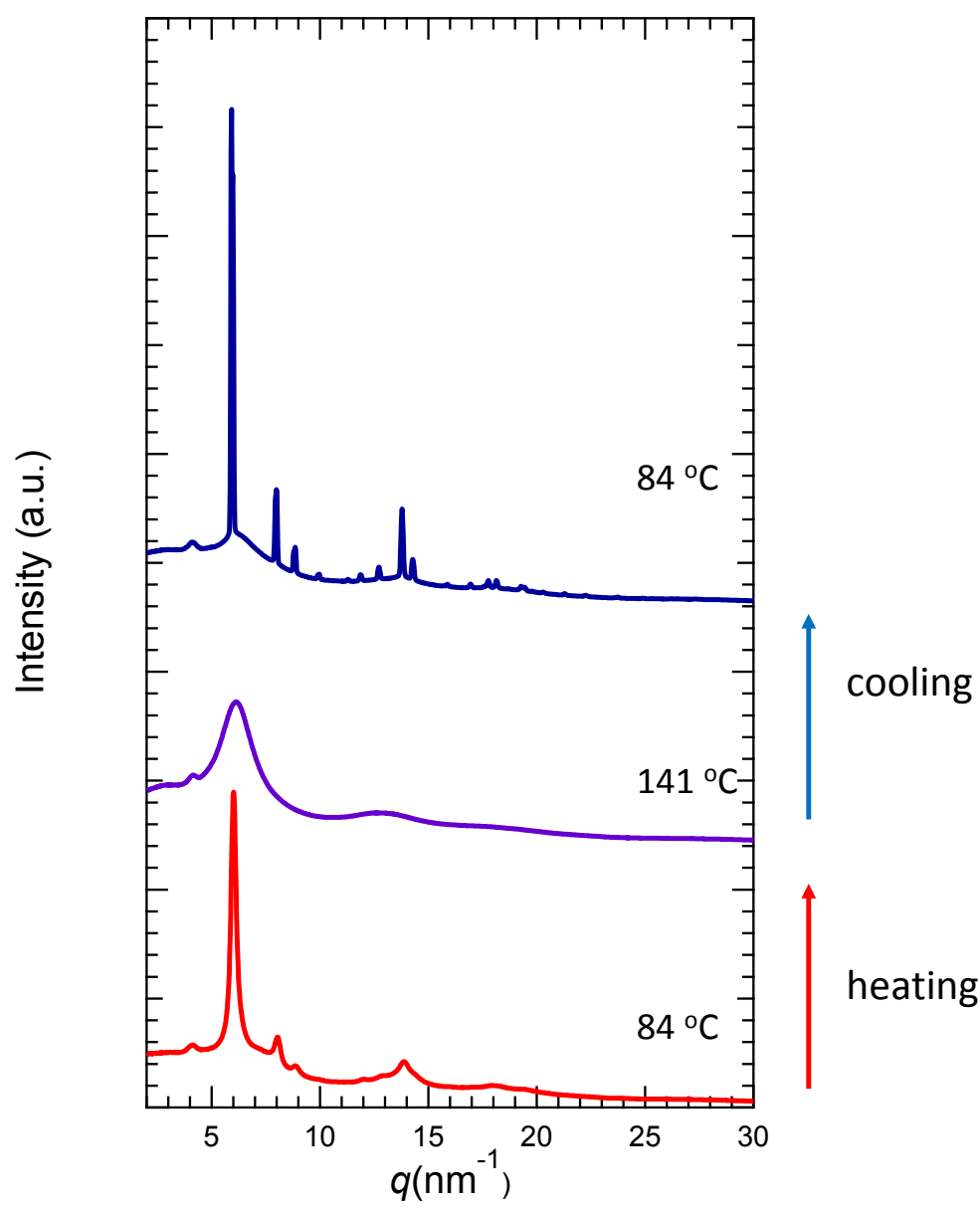

Figure S10. The scattering intensity is plotted against the scattering vector, $q$, for three temperatures for the neat POSS homopolymer. The WAXS pattern indicates the melting and recrystallization of POSS homopolymer. The peaks became sharper and more peaks appears on recrystallization. 
WAXS pattern of PEO homopolymer and kapton:
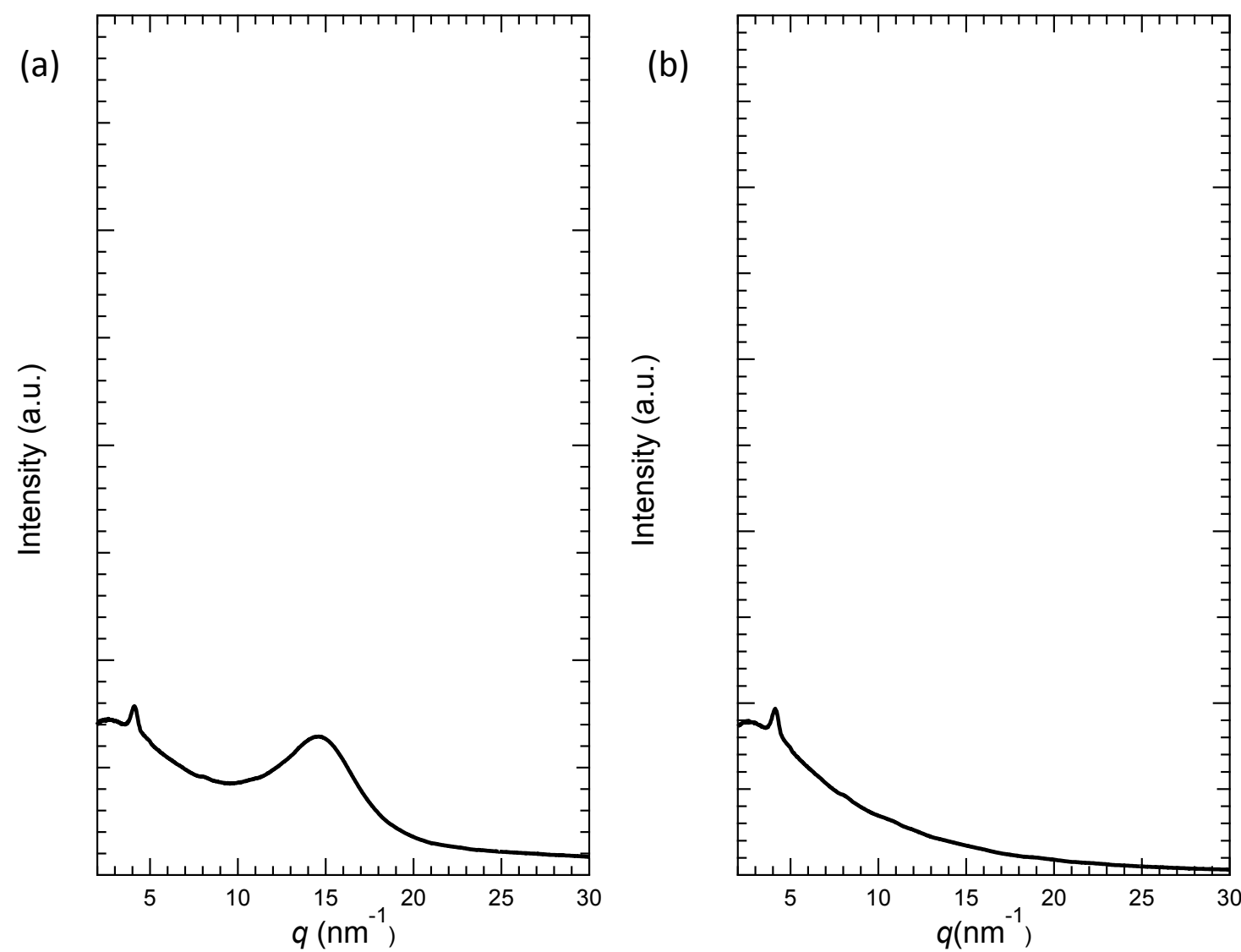

Figure S11. The scattering intensity is plotted against the scattering vector, $q$, for (a) PEO 10K homopolymer at $84{ }^{\circ} \mathrm{C}$, indicating amorphous nature of the polymer and (b) the blank kapton window. 
Conductivity of 6 individual cells:

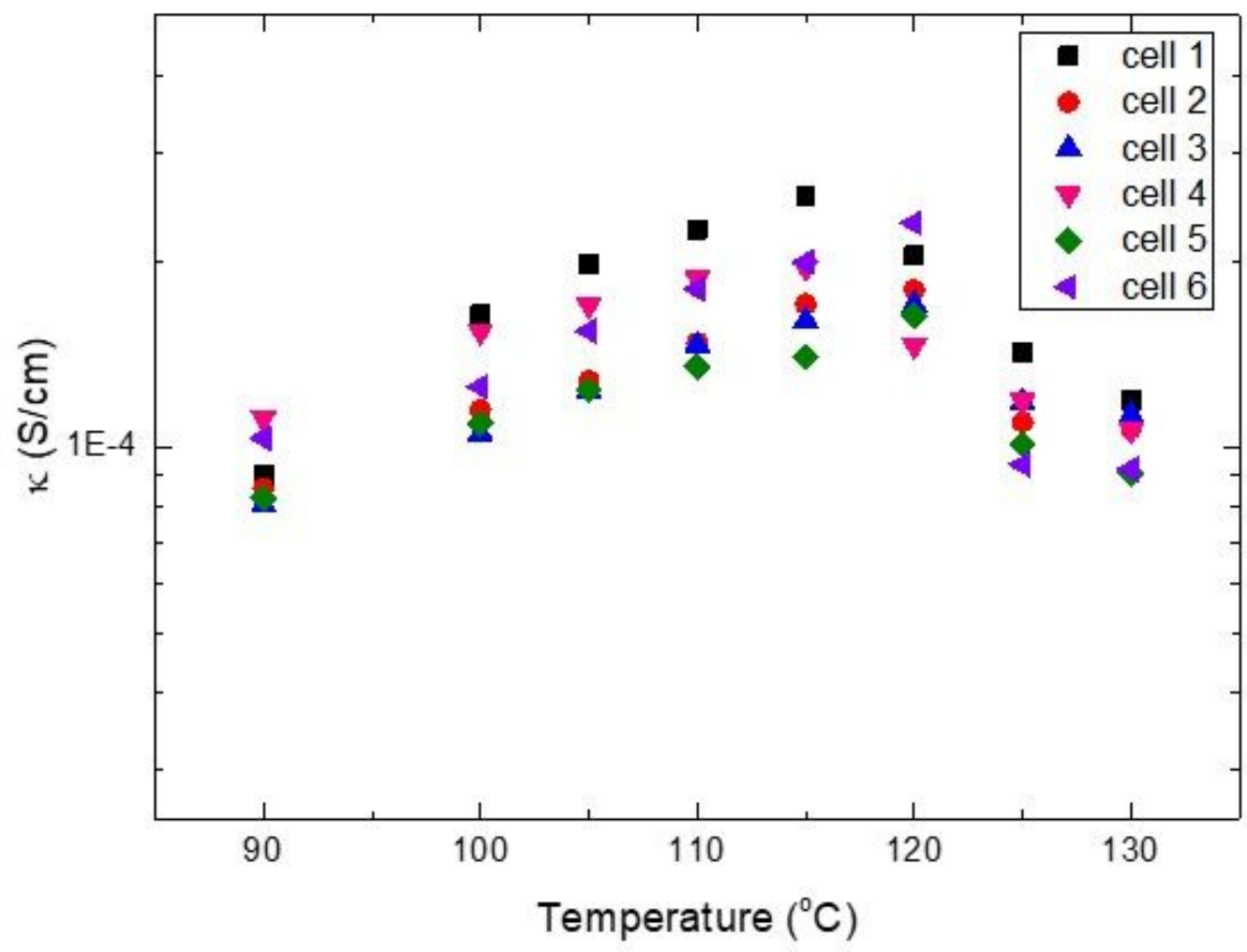

Figure S12. Conductivity value form six cells are plotted against temperature. The standard deviation is calculated from these values. 
Effect of annealing temperature on conductivity:

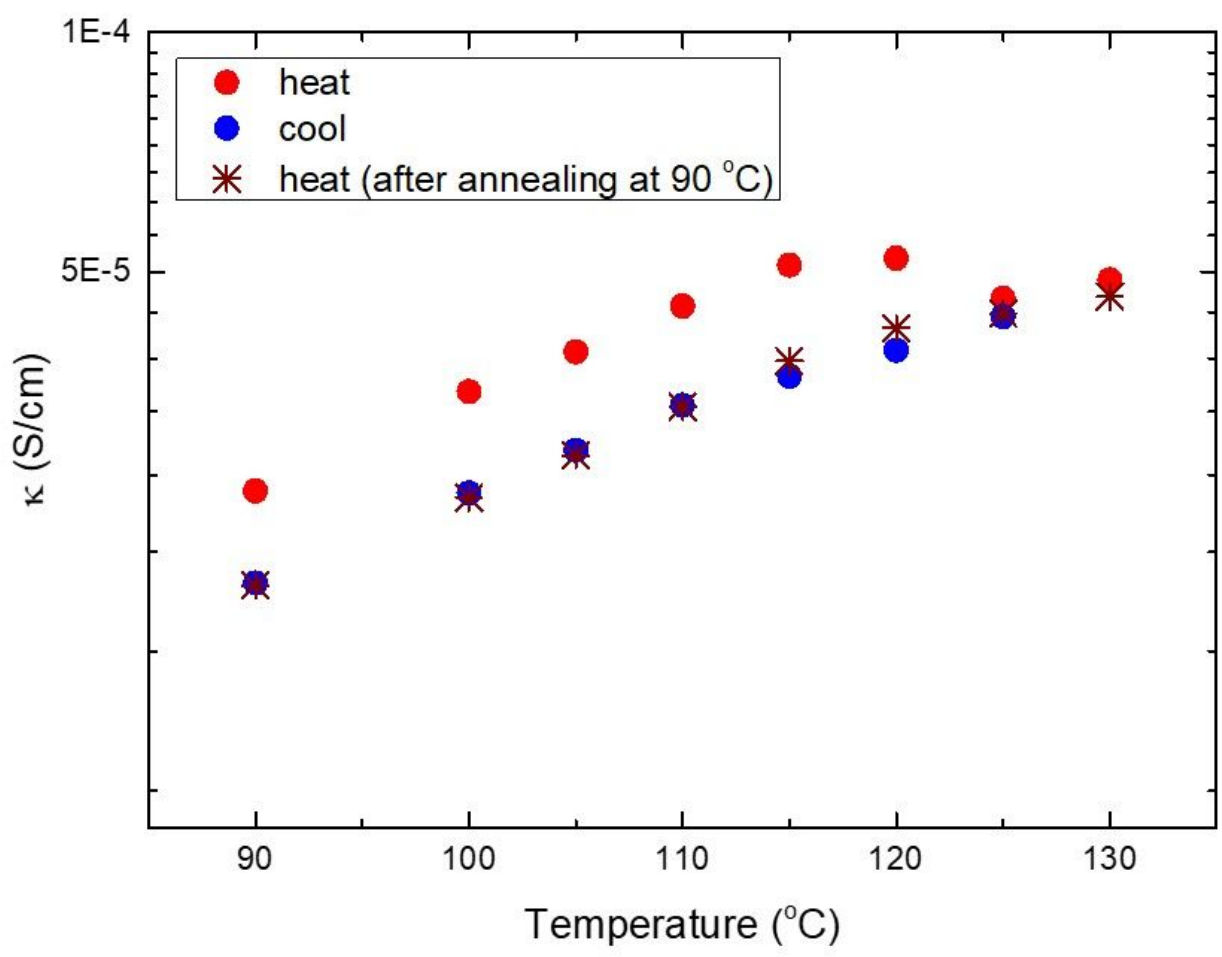

Figure S13. The temperature dependent conductivity of one of the cells is plotted. The red and blue circles represent the heating and cooling runs from first experiment and the maroon star represents the heating run after two days of annealing at $90{ }^{\circ} \mathrm{C}$. The second experiment represents that the conductivity does not recover after annealing the cell at $90{ }^{\circ} \mathrm{C}$. 